\title{
Pattern-Based Conceptual Modeling of Interaction with Cyber Physical Systems
}

\author{
http://dx.doi.org/10.3991/ijoe.v12i01.5200 \\ Junfeng Wang ${ }^{1,2}$, Suihuai $\mathrm{Yu}^{2}$, Ning Wang ${ }^{2}$ \\ ${ }^{1}$ Southwest University of Science and Technology, Mianyang China \\ ${ }^{2}$ Northwestern Polytechnical University, Xi'an China
}

\begin{abstract}
Supported by ubiquitous, pervasive and embedded computing, Cyber Physical Systems (CPS) support actors and groups by services anywhere and anytime. How users interact with CPS and the provided service is the key to success. To keep a holistic view of human-CPS interaction, we propose narratives, pattern-based diagrammatic conceptual models, and formalized conceptual models which are used for step-wise derivation of design models for distributed service infrastructures. Reusable design patterns for diagrammatic model are constructed for illustrating the human-CPS interaction processes. Finally, a use case is used to illustrate the implementation of the proposed method.
\end{abstract}

Index Terms-Cyber Physical System, System human interaction, conceptual models, design methodology, design patterns

\section{INTRODUCTION}

The development and integrating of mechanical systems, electromechanical systems, mechatronics systems, embedded systems, distributed real-time systems, and networked agent-based systems give birth to CyberPhysical Systems (CPS)[1]. As highly integrating computation with physical entities and processes, CPSs could penetrate into social world, even human mental world to provide human various services through interactions between human and system, subsystems, agents, devices and software, etc. CPSs feature heterogeneous components, adaptiveness, context awareness, distributed and decentralized, and multi-scale operation, etc. all of which differentiate interaction with it from traditional human computer/machine interaction. This is a new challenge for system developers and human-system interaction designers.

Design teams for Human-CPS Interaction are multidisciplinary; members from different fields such as information architects, domain experts, and various end users should be involved in design processes[2]. Ideas and expertise of these members have to be brought together for reaching agreements of an CPS. Approaches and tools that be used for communicating and explication are required for building common understandings. However, nontechnical members are prone to build an CPS that supports their own business, communication and social needs; technical members focus more on technical realization of the system. Such shared understandings of design teams can be described by various conceptual models (CM) that are used during design phases of CPS development [3].

Up to now, little research concentrated on dedicated design methodologies and conceptual modeling for Human-
CPS interaction[4]. Klemmer et al. explores CMs for designing tangible user interfaces based on physical input devices [5]. Janzen et al. propose a design methodology for Ubiquitous Information Systems that models situations by narratives and semi-structured representations [2] This paper tries to make the first steps in design methods for Human-CPS Interaction. After discussing the researches on interaction with CPS and CM, we will elaborate pattern-based conceptual modeling for interaction with CPS. Next, the processing of CMs by CPS is exemplified, and results and future work conclude this paper.

\section{RELATED WORK}

As more and heterogeneous actors, internal and external interrelationships involved in CPS, complexity of interactions with CPS much exceeds traditional Human Computer/Machine Interaction (see Fig. 1). Furthermore, to penetrate into real life process and environments, physical entities and process deeply merge with ubiquitous computing in CPSs, which interweaves human, devices, environments, cyber wares, and makes interaction more intricate[6]. There are four types of interaction should be designed: human-system interaction(HSI), System-human interaction(SHI), human-human interaction(HHI), and system-system interaction(SSI).

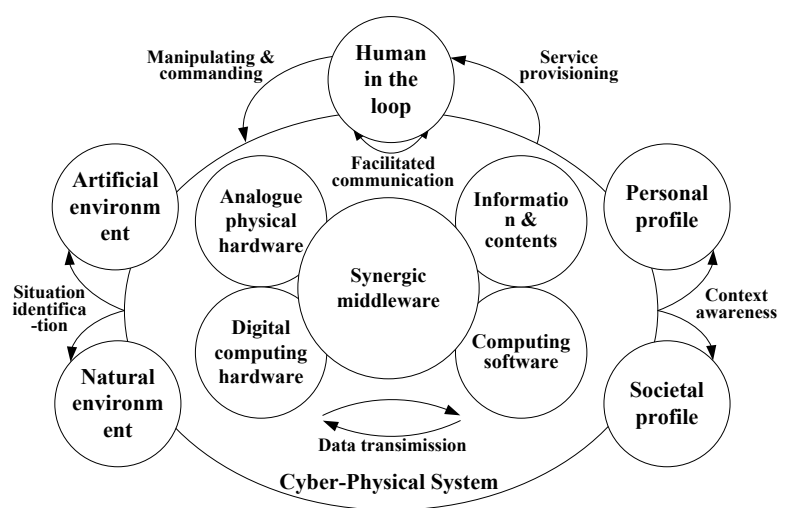

Figure 1. Interaction with CPS

There is no sufficient design methodology for interaction with CPS, we identify a methodology named "context-centered design of interaction with CPS(CCDIxC)" which considers the physical environment, cyberspace and users and the interaction process between two of them. It consists of five phases:(1)identifying problems, (2) identifying user needs, (3) designing solutions, (4) developing solutions, and (5) evaluating solutions. These phases consist of nine tasks: (1)identifying problems, (2) identifying 
contexts of use, (3) identifying user needs, (4) defining scenarios, (5) constructing functional architecture, (6)conceptual designing of system, (7) prototyping physical entities and cyber-ware and building a testing environment, (8) evaluating solutions, and (9) developing and deploying system.

On the other hand, conceptual modeling is a hot topic in the field of design science [7]. It focus on the identification of key concepts and relations that are semantically described by shared vocabularies [8]. These vocabularies are either implicitly defined as part of understanding in a community or explicitly defined in forms of representations that could be processed by machine[9]. CMs enable an abstraction from technical issues and focus on situations in which users perform activities that are grounded by information and communication services[3]. Conceptual modeling language (CML) is used to represent a CM. Entity-Relationship models and the Unified Modeling Language (UML) are common used CMLs. CMs could be used to analysis, design, and realize CPS during the development process.

Conceptual modeling for CPS deal with more complex requirements and intricate relationships between various components of the system. When users are moving in physical environments, system should provide service in seamlessly way with mobile or embedded device based on loosely coupled service infrastructures[10]. Hence, situated communication, collaboration of user groups and contextualized interaction in CPSs are far more complex than traditional HCI and HMI. It is highly dynamic, and context-dependent on various dimensions. This requires conceptual modeling articulate the logic and the required physical entities, information and cyber-ware of the interaction with CPS.

\section{CONCEPTUAL MODELING OF INTERACTION WITH} CPS

\section{A. Abstract model of CPS}

Conceptual models are strongly influenced by basic conceptualizations of a system[7]. In general, CPSs are compounds of physical entities, information, social systems, and service systems that use information technology infrastructures for realization of desired situations [11]. We bring up four conceptual classes for conceptual models of CPS:

- Physical world: all physical entities available within all operational situation of a CPS.

- Cyberspace: all information objects(IOs) used within the realm of an CPS.

- Social system: the set of roles available with a set of attributes, such as rights, obligations, and prohibitions, and actions performed by role-taking actors.

- Service system: the set of all digital and physical services available within all operational situation of a CPS.

Physical world encompasses all physical entities that are required to implement certain functionalities and fulfill certain service in CPS. Physical entities generate information related to itself, e.g. size, weight, and identity etc. All IOs are defined within an cyberspace based on supporting services. they are used in situations by the social system of role-taking actors. Actors useroles, IOs,

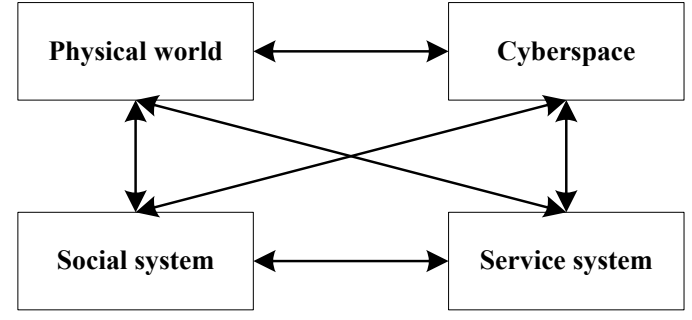

Figure 2. Abstract CPS Model

and services for certain goals in specific context. Interactions are explications of task requirements that are described by directed relations between roles. Communication is a sub-class of interactions. It transfers information that refers to IOs. IOs are abstract notions of all content in the forms of speech, written language, graphics, or digital representations.

Generally, a role-taking actor or a service provide supports for IOs. Furthermore, external services can create IOs and make them available to other services or roletaking actors. Services provide functions to roles and other services. Services that support roles are called interface services. Interface services provide graphical, tangible, speech or other interfaces by which role-taking actors access services for achieving some situation-specific goals.

\section{B. Three CMs}

Situations are use case based descriptions of interactions between entities in an environment. they use concepts and relations defined by shared vocabularies. CMs that are described by a CML capture situations and system architecture design ideas. The proposed method is based on three types of CMs: narrative CM, diagrammatic CM, and formalized CM.

Narrative CM depicts situations that address the defined problems or satisfy the needs that are identified together with domain experts in creativity workshops. Situations are textual descriptions of the status of physical and cyber entities, such as objects, roles, information, background environments and services. These entities perform particular activities and interact with each other and represent characteristic features of a corresponding class of situations within a domain, e.g., bathing situations. Thus, situations resemble interaction frames [12], schemas [13], and use cases. Narratives could provide discourse information and sequential orderings of interactions between actors. So, they are effective tools for building and understanding situations of future Information Systems [14]. In our case, we held a creativity workshop with a leading manufacturer of household appliance. With the help of several creativity techniques, situations, or more precisely narratives have been specified according to the question: "Where and how do we face information and media in our daily life?" The results were transformed into a textual description as follows:

"Jeffrey receives location based accommodation information from his smart phone when he is in travel."

Next, narratives are translated into diagrammatic CM. Based on the abstract CPS model, Diagrammatic CMs conceive usage situations by emphasizing requirements on social structure, IOs, physical entities, and services in a coherent structured manner. All core entities are identified in narratives that fit to these conceptual categories [4]. 
Similarly, relations are extracted that connect these entities.

To derive processable design patterns and specifications for system designs, diagrammatic CMs should be formalized $[15,16]$ by means of OWL. OWL ontologies could be read by machine, and thus is computational. Furthermore, OWL constructs are independent, i.e. classes can exist independent of instances or properties and properties are independent of classes [17]. A Diagrammatic $\mathrm{CM}$ represents a "vocabulary" and generic object proper- ties of Diagrammatic CM patterns [18]. Diagrammatic $\mathrm{CM}$ consists of concept types and generic object properties. It represents basic entities of Abstract CPS Model: $I O$, Role and Services with the sub-classes Interface and Internal Service. Furthermore, a super-class Action was defined that contains further sub-classes that specify diverse types of pattern actions: Creation, Receiving and Interaction with the sub classes R-Interaction and $S$ Interaction.

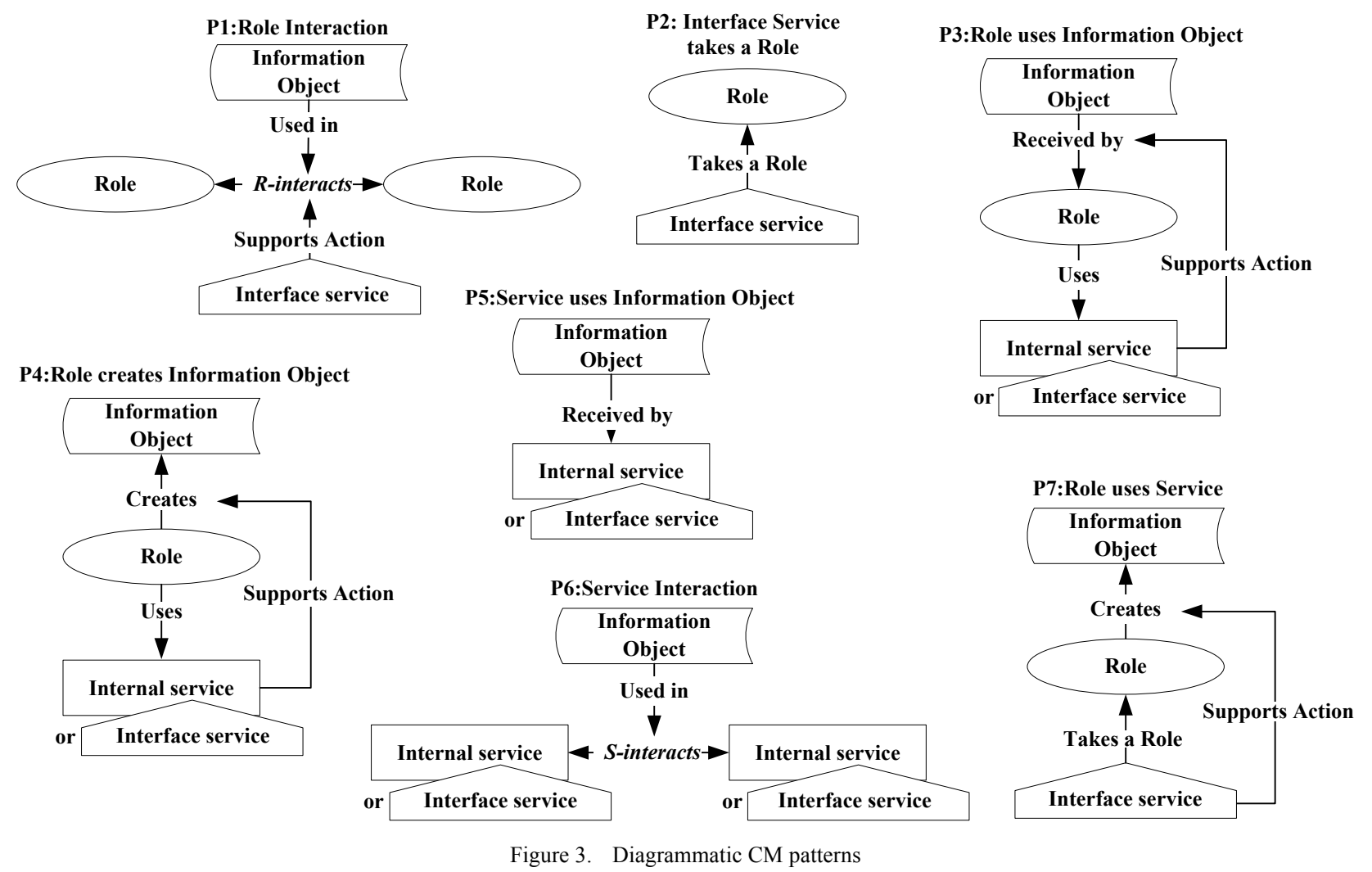

\section{Defining Diagrammatic CM with Patterns}

Analysis of Diagrammatic CMs in several Information System development projects showed re-occurring structures similar to the notion of design patterns as used in architecture [16] and Software Engineering [15]. They represent means for reusable CMs for Information System. We identified seven Diagrammatic CM patterns (see Fig. 3) which are elaborated as follows.

1) Role Interaction Pattern: This pattern describes a situation in which two or more role-taking actors interact with one another by exchanging IOs supported by an interface service, e.g., people communicate with each other by sending messages. Interaction between roles is described as r-interacts. The interface service is only used as a communication channel.

2) Service takes Role Pattern: This pattern expresses a situation in which a role is taken by an interface service. For example, government official websites release news and takes a role with social attributes, such as credibility and authenticity.

3) Service uses IOs Pattern: represents that an internal or interface service receives IOs without human interventions. This is a simplified Service Interaction pattern. It is used when a providing service is not important for a CM. For instance, accommodation information used by a travel assistant service is received from a cloud infrastructure.

4) Role creates Information Object Pattern: By this pattern a service creates information objects by taking a role which links an information object to a service. This pattern supports role-based system designs. For instance, a important sign monitoring system takes a role that allows it to create emergency alerts. Created alerts are directly linked with this service via a role.

5) Service Interaction Pattern: represents the interaction of two interface or internal services with no human actors interventing. This interaction is represented by $s$-interacts, and use information object. For instance, a local temperature service sends data to a central weather service. In contrast to the Role Creates Information Object pattern, this pattern supports system designs that do not use role-based on service level.

6) Role uses Service Pattern:This pattern describes a situation with a role-taking actor creating an information object. Therefore, the actor uses a service that supports the creation of an information object, e.g., a nurse who creates a status report for a patient by a healthcare reporting service. 
7) Role uses Information Object Pattern: In situations with direct manipulation of $I O s$, this pattern allows to express that a role receives an information object by using an internal or interface service. This means a roletaking actor can actively receive an information object supported by a service. For instance, a traveler uses a travel assistant service for accessing accommodation information.

Diagrammatic CM patterns provide conceptual structures as basic building blocks for CPS designs. The construction of Diagrammatic CM is guided by a method with five steps based on instantiation and integration of Diagrammatic CM patterns. (1) Defining IOs in Cyberspace, (2) Defining $H S I$ or/and $H H I$ related to $I O s$, (3) Defining Roles taken by Services, (4) Defining supporting Internal Service, and (5) Defining user initiative. Each step pursues a sub goal for constructing the Diagrammatic CM step by step and proclaims specific Diagrammatic CM patterns that help to achieve the objectives. In the following, we will exemplify the five steps with the exemplary narrative $\mathrm{CM}$ mentioned before.

\section{IMPLEMENTATION}

An example will be given for deriving a diagrammatic $\mathrm{CM}$ from a narrative $\mathrm{CM}$ based on the Diagrammatic CM, CML, Diagrammatic CM patterns, and CM method. Finally, the approaches of translating Diagrammatic CMs into propositional CMs are discussed and exemplified.

\section{A. Exemplary Defination of Diagrammatic CM}

Step 1: Defining IOs in Cyberspace. All information that occur in a narrative are defined as IOs in the Cyberspace. Fig. 4 shows this modeling step by means of an exemplary Diagrammatic $\mathrm{CM}$ that shall represent the narrative $\mathrm{CM}$ defined before. The figure shows that the modeling person has specified the goal "Getting user's location based accommodation information" that is assigned to the user in the situation. Furthermore, the information object location-specific accommodation information is defined. This information object has to be created in the situation based on the required information: local accommodation information and user's location.

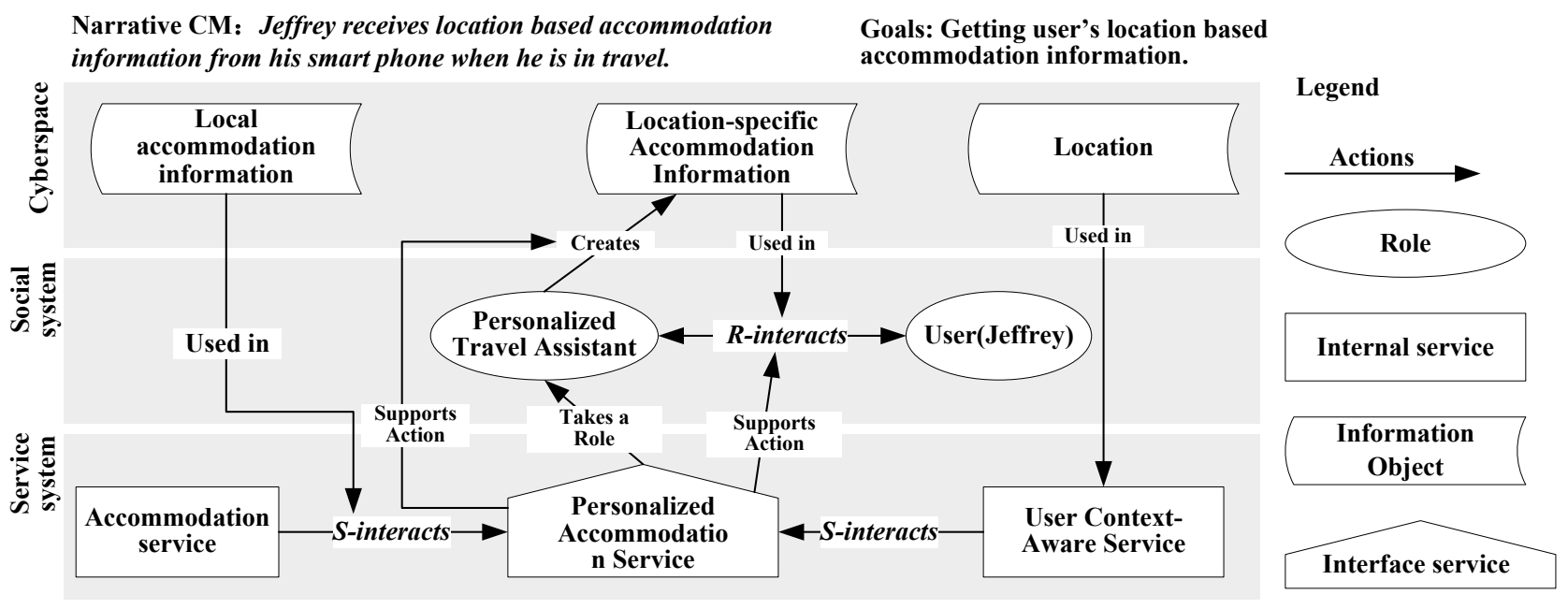

Figure 4. Derived Diagrammatic CM based on patterns

Step 2: Defining HSI or/and HHI related to IOs. Within this step, interactions between human or human and system related to newly generated IOs have to be defined. These interactions take place between Roles in Social System. This step connects Cyberspace and social world. HSI are always supported by a service of the Service System. This task is finished by applying the Role Interaction pattern. In the exemplary Diagrammatic CM (see Fig. 4) an interaction between a Personalized Travel Assistant and the User was modeled that is supported by a Personalized Travel Service. Subject of the interaction is locationbased accommodation information.

Step 3: Defining Roles taken by Services. Next, an interface service has to be defined. It creates new information object that will be used in the interaction while taking a role. Therefore, the service has to take a role in the interaction. To manage this step, the Role Creates Information Object pattern is applied to define the creation of the information object by a role taken by a service. In our example, the Personalized Travel Service takes the role of the Personalized Travel Assistant that creates the location-based accommodation information. The interface service supports this action indirectly (see Fig. 4). To express the plain role-taking by a service without a creat- ing function, the Service takes a Role pattern can be applied.

Step 4: Defining supporting Internal Services. To create new information objects, generic information sources are needed as mentioned before. The interface service that supports the creation of a new information object needs access to these sources. Therefore, internal services for all remaining information objects in the Cyberspace are specified. The interaction between services regarding the information objects is realized by applying the Service Interaction pattern. The exemplary Diagrammatic CM (see Fig. 4) shows the definition of two internal services Accommodation Service and User Context Service that feed a Personalized Travel Service with local accommodation information and location data.

Step 5: Defining user initiative. If a user role initiates an interaction with the system that means using the system in a proactive way, this situation is modeled by using the Role uses Service or Role uses Information Object pattern. This step is not required in this example because the user does not initiate interactions with the CPS (see. Fig. 4). 


\section{B. Exemplary Translation of Diagrammatic CM into Formalized CM}

To model the Formalized CM based on the diagrammatic CM, We use the expressiveness of Diagrammatic $\mathrm{CM}$ patterns represented as single ontologies. For derivation of propositional CM tools for modeling formal webbased representations are required, e.g. Protégé. After creating a new OWL file in Protégé, required patterns could be imported by their URL. Similar to the procedure of defining Diagrammatic CM in five steps, we start with applying the Role Interaction pattern and import its formalized model. Then, we instantiate the relevant concepts of the patter, i.e. we create instances of the concept "Role" named "User" and "Personalized Travel Assistant". To represent the interaction between User and Personalized Travel Assistant, we create an instance of the concept "Rinteraction". For linking both roles with the instance of RInteraction, the formalized pattern offers the specified object properties initatesR_Interaction and finalizesR Interaction that inherit from the super-properties initiatesInteraction and finalizesInteraction. Within the proceeding formalization, the formalized patterns Role uses $I O$ and Service Interaction are imported. Note, each pattern automatically imports the Diagrammatic CM. The result of the formalization is an OWL description that represents the exemplary narrative in a formal and computational way.

\section{CONCLUSION AND FUTURE WORK}

Recently, the development and design of CPS has been a hot topic. However, the interaction between users and CPSs gained less attention [19]. We introduced a pattern based conceptual modeling method for interaction with CPS. Three types of conceptual models - narrative, diagrammatic and formalized - are used and translated with in the design process of CPS. We exemplified all steps based a use case and elaborated the processing of the concept modeling by the resulting CPS. In the future, we will focus on verification of diagrammatic CM for designing CPS in other projects in comparison to other methods.

\section{REFERENCES}

[1] Horváth I. What the Design Theory of Social-Cyber-Physical Systems Must Describe, Explain and Predict?[M]//An Anthology of Theories and Models of Design. Springer London, 2014: 99120.

[2] Maass W, Janzen S. Pattern-based approach for designing with diagrammatic and propositional conceptual models[C]// Proceedings of the 6th international conference on Service-oriented perspectives in design science researchSpringer-Verlag, 2011:192206.

[3] Yair Wand, David E. Monarchi, Jeffrey Parsons, and Carson C. Woo. Theoretical foundations for conceptual modelling in information systems development. Decis. Support Syst., 15:285-304, December 1995. http://dx.doi.org/10.1016/0167-9236(94)00043-6

[4] Janzen S, Kowatsch T, Maass W. A Methodology for ContentCentered Design of Ambient Environments[J]. Lecture Notes in Computer Science, 2010:210-225. http://dx.doi.org/10.1007/9783-642-13335-0_15

[5] Scott R. Klemmer, James A. Landay. Toolkit Support for Integrating Physical and Digital Interactions[J]. Human-computer Interaction, 2009, 24(3):315-366. http://dx.doi.org/10.1080/073700209 $\underline{02990428}$
[6] Koskela T, V0101n01nen-Vainio-Mattila K. Evolution towards smart home environments: empirical evaluation of three user interfaces[J]. Personal \& Ubiquitous Computing, 2004, 8(3-4):234240. http://dx.doi.org/10.1007/s00779-004-0283-x

[7] Wand Y, Monarchi D E, Parsons J, et al. Theoretical foundations for conceptual modelling in information systems development[J]. Decision Support Systems, 1995, 15(4):285-304. http://dx.doi.org/10.1016/0167-9236(94)00043-6

[8] Lynne M. Markus, Ann Majchrzak, and Les Gasser. A Design Theory for Systems That Support Emergent Knowledge Processes. MIS Quarterly, 2002, 26(3):179-212.

[9] Maass W, Janzen S. Pattern-Based Approach for Designing with Diagrammatic and Propositional Conceptual Models[J]. Lecture Notes in Computer Science, 2011:192-206. http://dx.doi.org/10.1007/978-3-642-20633-7 14

[10] Lyytinen, K., Yoo, Y. Research Commentary: The Next Wave of Nomadic Computing. Information Systems Research 2002, 13(4), 377-388. http://dx.doi.org/10.1287/isre.13.4.377.75

[11] Xing J Y, Zhang L C, Software S O. Cyber Physical System Modeling Based on Energy Control and Resource Scheduling[J]. Computer Technology \& Development, 2014.

[12] Minsky,M. A framework for representing knowledge. Technical report, Cambridge, MA, USA, 1974.

[13] Schank, R.C., Abelson, R.P. Scripts, Plans, Goals and Understanding: an Inquiryinto Human Knowledge Structures. L. Erlbaum, Hillsdale, 1977.

[14] Kuechler, W., Vaishnavi, V. On theory development in design science research: anatomy of a research project. European Journal of Information Systems 2008,17, 489-504. http://dx.doi.org/10.1057/ejis.2008.40

[15] S. Purao, T. Han, and V. Storey. Improving Reuse-based Design: Augmenting Analysis Patterns Reuse with Learning. Information Systems Research, 2003, 14(3):269-290. http://dx.doi.org/10.1287/isre.14.3.269.16559

[16] P. Bera, A. Krasnoperova, and Y. Wand. Using Ontology Languages for ConceptualModeling. Journal of Database Management, P. Bera, A. Krasnoperova, and Y. Wand. Using Ontology Languages for Conceptual Modeling. Journal of Database Management, 21(1):1-28, 2010, 21(1):1-28. http://dx.doi.org/10.4018/ jdm.2010112301

[17] Rossi, M., Sein, M.K.: Design research workshop: A proactive research approach,2003.

[18] Rouge C L, Niedeman F. information systems and health care xi: public health knowledge management architecture design: a case study[J]. Communications of Ais, 2006

[19] Youngjin Yoo. Computing in Everyday Life: A Call for Research on ExperientialComputing. MIS Quarterly, 34(2):213-231, 2010

\section{AUTHORS}

Junfeng Wang (Corresponding author) and Wenjun Wang are with the school of Manufacturing Science and Technology, Southwest University of Science and Technology, Mianyang Sichuan 621010, China (email:wjfchi@foxmail.com).

Suihuai Yu is with the Institute of Industrial Design, Northwestern Polytechnical University, Xi'an 710072, China (email:ysuihuai@vip.sina.com).

This work was partially supported by Chinese Scholarship Council (Grant No. 20135044), National Key Technology R\&D Program of China (Grant No. 2015BAH21F01), Xi'an Key Technology R\&D Program of China (Grant No. FW1409), and R\&D Program of Sichuan Key laboratory of manufacturing process testing technology (Grant No. 13zxzk05). Submitted 06 November 2015. Published as resubmitted by the authors 27 December 2015. 